\title{
CAMBIOS RECIENTES EN LOS SERVICIOS SOCIALES PARA MENORES EN INGLATERRA
}

\author{
M. ${ }^{a}$ ASUNCIÓN MARTÍNEZ ROMÁN* \\ Departamento de Trabajo Social y Servicios Sociales. Universidad de Alicante.
}

\section{INTRODUCCIÓN}

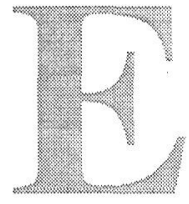

1 modelo de «Estado de bienestar» británico se establece a partir de 1940, cuando el Estado asume la responsabilidad pública en la atención de las necesidades sociales. Se crea el Sistema Nacional de Salud, se universaliza la educación, se diseña el sistema de Seguridad Social, las organizaciones caritativas dejan de ser las principales proveedoras de servicios sociales personales y se asume también la intervención pública en materia de alojamiento ${ }^{1}$. Tras la Segunda Guerra Mundial este modelo se desarrolla y consolida, coincidiendo con una época de crecimiento económico, como resultado de una política de consenso de los principales partidos políticos.

En cuanto a los Servicios Sociales, hay que destacar que han venido siendo uno de los pilares del modelo de Estado de Bienestar en el Reino Unido, habiendo experimentado un desarrollo creciente con sucesivas reformas en su concepción, organización y gestión. Las propuestas del Informe Seebohm ${ }^{2}$ inspiraron la Ley de Servicios Sociales de 1971 por la que se reorientaron los servicios sociales llevándose a cabo su descentralización al gobierno local. El nuevo modelo abandonó la excesiva especializacion anterior promoviendo una atención social más generalista, tendencia que se reorientará de nuevo en los 80 recuperando la apuesta por la especialización en los servicios para la atención de menores y de personas con enfermedades mentales.

El país se encuentra administrativamente dividido en ciscunscripciones a cargo de Autoridades Locales que se constituyen en «conda-

* Agradezco a mi colega Carlos Fernández (Trabajador Social, Doncaster, UK) la valiosa ayuda prestada con la revisión de este trabajo y sus muy oportunas sugerencias, tal como viene haciendo (con paciencia y cariño) desde 1993.

Hills, J. (1990): The State of Welfare. Oxford, Clarendon Press.

2 Home Office (1968): Report of the Committee on Local Authority and Allied Personal Social Services. (Cm 3703). Londres, HMSO. 
dos» (County Council) o en «distritos metropolitanos» (London Boroughs y Metropolitan Districts, que incluyen las grandes ciudades y las áreas que las circundan). Los County Council, London Boroughs y Metropolitan Districts tienen las mismas competencias para proporcionar servicios a través de sus Departamentos de Servicios Sociales.

Estos Departamentos de Servicios Sociales de la Administración local dependen del Ministerio de Salud (Department of Health), desde el que se diseña la política y planificación de los Servicios sociales asegurando la integración de la planificación entre los servicios de salud y los servicios sociales. Además, se financia el gasto social global de la administración local y cada autoridad local puede decidir la distribución de esa financiación; se establecen criterios de actuación de los servicios sociales; y se realiza la inspección y evaluación de los servicios (Social Service Inspectorate).

$\mathrm{El} \ll$ Home Office» es el Ministerio con competencias relacionadas con la ley de la que depende la atención a menores con problemas de delincuecia y que trabaja en estrecha coordinación con los Departamentos de Servicios Sociales locales. También ha cofinanciado con la Unión Europea la creación de servicios sociales comunitarios en grandes ciudades como, por ejemplo, servicios de guardería para menores, centros de día para mayores, unidades residenciales para menores, o servicios para personas con discapacidades. Otro Ministerio que trabaja en coordinación con los Departamentos de servicios sociales locales es el Ministerio de Educación y Empleo.

Pero es en el nivel local en donde se encuentra la mayor responsabilidad en materia de Servicios Sociales, siendo los Departamentos de Servicios Sociales de cada County los encargados de prestar servicios tanto de tipo preventivo, dirigidos a toda la población, como servicios para atender a colectivos específicos:

- Cuidado de personas mayores en la comunidad y residencias.

- Cuidado de personas con discapacidad física.

- Orientación y consejo a homeless.

- Servicios de cuidado de menores (protección de menores, residencias, guarderías, acogimiento familiar, algunos servicios de adopción, trabajo preventivo con maltrato de menores, etc.).

- Trabajo social con personas con enfermedades mentales y sus familias, incluyendo servicios de centros de día, talleres y alojamiento.

- Cuidados de día para niños menores de 5 años, incluyendo guarderías y supervisión de guarderías privadas. 
- Ayuda y comida a domicilio.

- Cuidado de madres solas, incluyendo residencia.

- Trabajadores Sociales para personas ingresadas en hospitales.

- Trabajo con abuso de sustancias (droga y alcohol).

Organizativamente, los Departamentos de Servicios Sociales locales se basan en diferentes modelos, por ejemplo, un Director como responsable de la administración de todos los servicios, auxiliado por Asistentes de dirección. Se establece una división por Distritos y, dentro de cada uno de ellos, se establece una subdivisión de los equipos según se trabaje con menores o con adultos, lo que implica una especialización. Deben coordinarse estrechamente con otros profesionales, como visitadores de salud, enfermeras comunitarias/de distrito, trabajadores sociales sanitarios, médicos, profesores, trabajadores sociales psiquiátricos, trabajadores de comunidad, líderes juveniles y voluntariado social de la zona.

Entre las actividades que se realizan en los Departamentos cabe citar las siguientes:

- Diagnóstico y evaluación de necesidades. Tras la evaluación de necesidades se puede ofrecer la prestación de un servicio bien de la propia Autoridad Local o bien "comprando" el servicio a una entidad privada sea no-lucrativa o mercantil.

- Trabajo de campo: atención a menores y familias, mayores, personas con discapacidades, transporte, ayuda a domicilio, servicios de comidas, guardería, familias sin techo, servicios de adopción.

- Actividades de formación: formación inicial para nuevos incorporados, formación continua, formación profesional especializada, formación para la docencia de alumnos en prácticas, formación para dirección de residencias, formación post-graduada senior para puestos de responsabilidad y dirección-gestión.

- Trabajo social hospitalario, para ayudar a las personas ingresadas y a sus familias a convivir con la enfermedad

- Actividades de elaboración de informes y cumplimentación de formularios de diversa índole exigidos por la normativa vigente, por ejemplo, toda la elaboración de Informes para el Juzgado que establece la Ley de Menores.

En cuanto a la financiación, el gobierno central establece un patrón de gasto tipo (Standard Spending Assessments) por el que se estima el gasto que precisa realizar una Autoridad Local para proporcionar un nivel básico de servicios, teniendo en cuenta las diferentes característi- 
cas demográficas, sociales, económicas, meteorológicas y geográficas del territorio ${ }^{3}$.

\section{LOS SERVICIOS SOCIALES EN INGLATERRA DURANTE LOS $80 / 90$}

Durante la crisis económica de mediados de los 70 se empieza a cuestionar el aumento del gasto social de los servicios sociales públicos y a lo largo de los años 80 el gobierno conservador de Mrs. Thatcher llevó a cabo importantes cambios en el modelo de servicios sociales, tanto en el modo de distribución como en los principios subyacentes ${ }^{4}$, promoviendo un cambio de valores que incluía la aceptación de la existencia de la desigualdad social como un hecho inevitable y asignando un papel relevante al mercado. Las políticas de los 80 se basaron en la idea de un Estado mínimo que cedía el protagonismo a la iniciativa privada, potenciando la centralización con una reorientación de las competencias de las autoridades locales, en detrimento de su autonomía (reducción de independencia financiera y limitación de su rol y funciones) $)^{5}$ en áreas relacionadas con el bienestar como las de salud, educación, vivienda y servicios sociales ${ }^{6}$.

La resistencia a romper el anterior modelo de servicios sociales públicos por parte de los laboristas dio lugar a diferencias territoriales en la oferta de servicios sociales, ya que algunas Autoridades Locales se resistieron a aplicar las nuevas políticas basadas en la drástica reducción del gasto público, reducción de los subsidios, el incremento de las prestaciones sujetas a la demostración de necesidad y la privatización de los servicios. El desarrollo de la intervención del sector mercantil se generalizó con la aplicación en 1991 del plan de cuidados en la comuni$\mathrm{dad}^{7}$ que disminuye el papel del Estado y promueve una mayor intervención de la ayuda informal y de la iniciativa privada, tanto no-lucrativa como mercantil.

3 Carr-Hill, R.; Rice, N.; Smith, P. (1999): "The Determinants of Expenditure on Children's Personal Social Services". British Journal of Social Work, 29, 679-706.

+ Johnson, N. (1991): Reestructuring the Welfare State. Hemel Hempstead, Harvester Wheatsheaf.

5 Young, H. (1989): One of Us. Londres, Macmillan, págs. 124-125.

6 La «Education Reform Act» de 1988, transfiere importantes competencias desde el gobierno local hacía el gobierno central. La «Local Government Act» de 1988, tuvo especial relevancia en este vaciado de poder de los gobiernos locales a favor de una mayor centralización.

7 Department of Health (1989): Caring for People (Cm 849), Londres, HMSO. 
Diversos autores coinciden en señalar que las políticas de los 80-90 ha incrementado la pobreza y la desigualdad y que muchas personas, además de carecer de medios económicos, han tenido negado el acceso a la mayoría de las actividades y servicios. La consecuencia ha sido una falta de oportunidades para ellos y también para sus hijos y, en definitiva, una menor calidad de vida que el resto de la población ${ }^{8}$, pagando un alto costo personal: malnutrición, preocupación cada día por conseguir ingresos y situación permanente de endeudamiento? . En estas familias pobres los niños son los miembros más vulnerables con una alta mortalidad, bajo peso al nacer, más posibilidad de discapacidades, caries dental, bajo crecimiento y malnutrición ${ }^{10}$.

En el origen de esta situación de empobrecimiento se encuentran distintos factores como el aumento del desempleo, los cambios en el empleo (trabajo temporal, ocasional, autoempleo...), que reducen los ingresos, cambios de las pautas familiares (aumento de la monoparentalidad), inadecuada respuesta del sistema de protección social (diseñado para una situación de pleno empleo), etc. ${ }^{11}$ Según Craig, la tendencia general en la composición de la pobreza en UK, como en muchos países de la Comunidad Europea, ha sido pasar de una concentración en las personas mayores hacía la extensión de la pobreza a una serie de diferentes categorías o grupos de personas como: personas desempleadas (especialmente de larga duración), familias con niños, padres/madres solos, personas con escasos ingresos y personas con discapacidad ${ }^{12}$.

\subsection{Infantilización de la pobreza}

Veamos más detenidamente cómo las consecuencias del aumento de las situaciones de pobreza y exclusión social han afectado en gran medida a los menores, especialmente, a aquellos que formaban parte de hogares con bajos ingresos o de hogares monoparentales. Estos menores han estado viviendo en una situación de permanente desventaja que ha mermado sus posibilidades de desarrollo.

Los estudios muestran que ha habido un notable incremento del número de padres/madres solos con hijos menores (en la mayoría de los

s National Children's Home (1991): Children in danger 1991: Family nutrition survey, Londres, $\mathrm{NCH}$.

9 Bradshaw, J. y Millar, J. (1991): Lone-parent fanilies in the UK. Londres, HMSO; Payne, S. (1991): Women, Health and Poverty. Haverster Wheatsheaf.

to Bradshaw, J. (1990): Child Poverty and deprivation in the UK. Londres, National Children's Bureau.

" Oppenheim, C. (1993): Poverty: The Facts. Londres, CPAG, págs. 2-3.

12 Craig, G. (1992): «Anti-Poverty Action and research in the U.K.», Social Policy \& Administration, vol. $26, \mathrm{n}^{\circ}$ 2. 131 . 
casos es la mujer la cabeza de familia, que no disponen de medios suficientes para atender las necesidades del hogar, generando stress y falta de oportunidades para los padres/madres y sus hijos ${ }^{13}$. En 1993, los hogares monoparentales tenían la mitad de los ingresos que las parejas; los más pobres de los hogares monoparentales estaban formados por mujeres solteras, seguidos de los formados por mujeres separadas. Según Haskey, la edad media de las madres solas con hijos a cargo era de menos de 25 años para las solteras y de 43 años para las divorciadas $^{14}$.

Uno de los problemas de estas madres ha sido (y continúa siendo) la falta de servicios para cuidar a los niños menores de cinco años, lo que resulta un grave obstáculo al trabajo de las madres, con el agravante de que sobre un $40 \%$ de estas madres no han tenido nunca un empleo ${ }^{15}$. Este hecho, junto con la alta probabilidad que tienen de no encontrar más que trabajos inseguros y mal pagados, explica que manifiesten preferir quedarse en casa, cuidando a sus hijos ${ }^{16}$. Millar dibuja el siguiente perfil de las familias monoparentales en 1989: mujer joven (menos de 25 años), con hijos menores a su cargo, que se aloja en una vivienda social de alquiler, con malas condiciones de habitabilidad, carece de empleo y es beneficiaria de una prestación económica pública ${ }^{17}$. Con relación a la vivienda, se ha denunciado que las familias monoparentales han venido teniendo baja prioridad en los baremos de adjudicación de viviendas sociales municipales, por lo que han tenido que conformarse con los peores alojamientos, incluso obtener simplemente la modalidad $\ll$ bed \& breakfast» $\mathrm{y}$, al tener mayoritariamente muy bajas rentas, no han podido optar a otras modalidades mejores de alojamiento público ${ }^{18}$. Se constata, también, una alta probabilidad de enfermedades y discapacidades en estos padres/madres y sus hijos ${ }^{19} \mathrm{y}$, especialmente, en aquellos alojados en la modalidad de «bed \& breakfast».

Los datos son elocuentes, tal y como lo muestra la tabla con el nú-

13 Algunos estudios señalan que el stress generado por graves carencias económicas aumenta la probabilidad de que los padres maltraten a sus hijos. Ver, por ejemplo, las consecuencias en el caso de Ias madres solteras, Gorlic, C. (1988): Economic stress, social support and female single parents. Canadian Social Work Review, vol.5, págs. 194-205.

if Haskey, J. (1991): Estimated numbers and demographic characteristics of one parent families in Great Britain. Population Trends, n 65, págs. 35-47.

15 Marsh, A. y McKay, S. (1993), op. cit., pág. 60.

16 Jowcll, R. et al. (1993): Bristish Social Attitudes: the 9th. Report. Aldershot, Dartmouth.

17 Millar, J. (1989), op. cit., pág. 68.

18 Sexty, C. (1990): Women Losing Out: Access to Housing in Britain Today. Londres, Shelter, págs. 33-35.

19 Hardey, M. y Crow, G. (Eds.) (1991): Lone Parenthood: Coping with constraints and making opportunities. Hemel Hempstead, Harvester Wheatsheaf. 
Tabla 1: Niños en familias en o por debajo del ingreso mínimo. Reino Unido, 1979 , 1989 y 1992

\begin{tabular}{|l|l|r|r|r|}
\cline { 2 - 5 } \multicolumn{2}{c|}{ Con sus padres } & $\mathbf{1 9 7 9}$ & 1989 & 1992 \\
\hline \multirow{2}{*}{ Familia monoparental } & Total & 840 & 1,350 & 1,850 \\
\cline { 2 - 5 } & \% de la categoría & 7 & 13 & 18 \\
\cline { 2 - 5 } & Total & 660 & 1,410 & 1,840 \\
\hline
\end{tabular}

Fuente: CPAC, Poverty: The facts. Eds. 1993 y 1996. Elaboracion propia.

mero y porcentaje de niños viviendo en familias que se encuentran en el nivel del ingreso mínimo o por debajo de él. Como puede verse, los niños que estaban en tal situación eran, en 1979, un $7 \%$ de los niños que vivían con sus padres y un $47 \%$ de los que vivían en familia monoparental. En cambio, en 1992, el porcentaje ha pasado, respectivamente, a un $18 \%$ y a un $78 \%$, es decir, que el $78 \%$ de los niños que vivían en esa fecha en una familia monoparental lo hacían en una familia de renta inferior o igual al ingreso mínimo, o sea, consideradas como pobres.

Esta «infantilización» de la pobreza queda insistentemente reflejada en todos los trabajos consultados, donde se muestra fundada preocupación por las consecuencias a corto y largo plazo de este problema. La tabla hace ver cómo el porcentaje de niños viviendo en familias con la mitad de la renta media (definición habitual en la Unión Europea) es superior al de la población total. Los datos de 1992/1993, sugieren que un cuarto de la población vive en la pobreza (definida como se ha dicho) de la que los niños constituyen un tercio.

Los propios Departamentos de Servicios Sociales locales han señalado como indicadores de ausencia de bienestar infantil las situaciones de familias «sin techo», alojamiento en «bed \& breakfast», en viviendas infraestandard y carencia de gas, electricidad y/o agua; considerando entre los factores de riesgo el pertenecer a una familia de bajos ingresos, monoparental, o de padres desempleados junto a otros factores como ser hijo de refugiados, pertenecer a una minoría étnica o lingüística, vivir en determinadas áreas, etc. ${ }^{20}$

Tabla 2: Porcentaje de niños y de población viviendo con la mitad de la renta media (descontados gastos de vivienda). Reino Unido, 1979 a 1992/93.

\begin{tabular}{|l|r|r|r|r|c|}
\cline { 2 - 6 } \multicolumn{1}{c|}{} & $\mathbf{1 9 7 9}$ & $\mathbf{1 9 8 1}$ & $\mathbf{1 9 8 7}$ & $\mathbf{1 9 8 8 8 9}$ & 199293 \\
\hline Niños & 10 & 16 & 24 & 25 & 33 \\
\hline Población & 9 & 11 & 19 & 22 & 25 \\
\hline
\end{tabular}

Fuente: CPAC, Poverty. The facts. Eds. 1993 y 1996. Elaboracion propia. 
Y unos años después, al finalizar el siglo, la situación no ha mejorado. El más reciente estudio publicado sobre la pobreza y la exclusión social en Gran Bretaña ${ }^{21}$ destaca que, a finales de 1999, un $26 \%$ de la población vivía en situación de pobreza, medida en términos de bajos ingresos y falta de satisfacción en varias de sus necesidades. Se estima que dos millones de menores tienen necesidades no cubiertas siendo peor la situación de los menores que viven en hogares con las siguientes características familiares: estar ambos padres desempleados; tener un solo progenitor; tener miembros de la familia sufriendo enfermedades crónicas; pertenencia a una minoría étnica; habitar una vivienda social; depender económicamente de una prestación de desempleo o una prestación asistencial.

En definitiva, siguen existiendo situaciones de pobreza y exclusión social que afectan en mayor medida a los grupos sociales más vulnerables y, entre ellos, a los menores. Ello se debe, por una parte, a un aumento del desempleo que ha evolucionado a una larga duración o a la precarización del empleo y, por otra parte, a un recorte de los gastos sociales que ha limitado las ayudas públicas para proteger a los más débiles. Tanto el desempleo como el recorte del gasto social público han sido factores comunes en la Unión Europea, sin embargo, en el caso de Gran Bretaña la disminución de la protección social pública ha sido mayor debido a las políticas de los gobiernos conservadores basadas en un Estado mínimo, arguyendo que las prestaciones públicas generan dependencia. Esta orientación política incidió muy directamente en los Departamentos de Servicios Sociales de las Autoridades Locales.

\subsection{Consecuencias en los Servicios Sociales y en la atención so- cial a los menores}

En este contexto de Estado mínimo, los Departamentos de Servicios Sociales locales tuvieron que adoptar nuevas líneas de actuación pasando de prestar directamente los servicios a evaluar e identificar las necesidades para proponer nuevas formas de atención, en la línea del Welfare Mix. Las nuevas modalidades de prestación de los servicios se pueden realizar por una variedad de agencias (con una mayor participación del sector voluntario), entre las que los Departamentos de Servi-

Giller, H. (1993): Children in need: Definition, Managment and Monitoring. A report for the Department of Health. Londres, Social Information Systems-Department of Health; Algate, J. et al. (1994): Implementing Section 17 of the Children Act. The first 18 months. Leiccster, Leicester University.

21 Gordon, D. et al. (2000): Poverty and social exclusion in Britain. York, Joseph Rowntree Foundation. 
cios Sociales locales han pasado a ser uno más, con una fuerte privatización de los servicios sociales públicos. Las consecuencias han sido importantes, por ejemplo, introduciendo la competencia entre los servicios de atención comunitaria para cuya prestación se han constituido consorcios de agencias de cuidados, en las que participan el Servicio Nacional de Salud, el gobierno local y el sector voluntario. Además, a muchos usuarios se les exige contribuir económicamente en el costo de los servicios, por lo que muchas personas no pueden utilizarlos, obligando a algunas a recurrir a cuidados residenciales ${ }^{22}$.

Se fueron reduciendo los recursos públicos, dirigiendo su atención a «los que más lo necesitaban» (con diferentes interpretaciones de «necesidad»), exigiendo una importante participación en los cuidados a las familias y comunidades y atendiendo prioritariamente a la población que carece de apoyo familiar, lo que supone que se han dejado de atender muchos problemas. Los Trabajadores sociales continuaron teniendo un papel muy relevante, pero con un nuevo rol que les asignaba funciones de gerentes y organizadores de los servicios más que realizar trabajo social.

En el caso de la atención social a los menores, la aplicación de la Ley del Menor de 1989 ha supuesto una serie de mejoras en los servicios sociales para menores, sin embargo, también se han puesto de manifiesto una serie de temas no resueltos y a los que la actual reforma trata de encontrar solución, por ejemplo:

- Heterogeneidad territorial de la oferta.

Ha habido una oferta heterogénea en cuanto a cantidad y calidad de servicios como consecuencia de la autonomía de las autoridades locales ${ }^{23}$, lo que ocasiona desigualdades en la atención de los menores.

- Protección en detrimento de la promoción del bienestar.

Se ha seguido dando prioridad a la «protección» de los menores entendida ésta en un sentido muy restrictivo, como actuaciones ante un riesgo considerable de maltrato o abuso. Generalmente, no se han dedicado esfuerzos a intervenir de modo preventivo para evitar el maltrato o el abuso, por ejemplo, actuando ante problemas que pueden tener consecuencias tan graves para bienestar del menor como el hecho de vivir en hogares pobres o con problemas escolares, cuando está demostrada la interrelación

22 Housc of Commons Health Committee (1993): Sixth Report of the Health Committee. Community Care: The Way Forward. Vol. 1. Londres, HMSO.

23. Department of Health. Social Services Inspectorate (1990): Child Care Policy: Putting it in wrinting. A Review of English Local Authorities' Child Care Policy Statements. Londres, HMSO. 
entre pobreza, privación y necesidad de intervención para proteger al menor ${ }^{24}$.

- Deficiente seguimiento de los menores.

Se han detectado actuaciones de los Departamentos de Servicios Sociales en las que no se realiza un adecuado seguimiento que permita evaluar periódicamente el bienestar del menor. En consecuencia, muchos problemas de los menores no se cono$\operatorname{cen}^{25}$ e, incluso, se han dado casos de abuso y maltrato de menores mientras éstos estaban bajo el cuidado de las Autoridades Locales.

- Insuficiente oferta de servicios de apoyo a las familias y cuidadores de personas dependientes.

La oferta de servicios para menores se ha considerado insuficiente para prevenir dificultades de los menores en su desarrollo con quejas de falta de financiación por parte de las Autoridades Locales. Esta escasez de recursos económicos es la excusa para actuar muy selectivamente, lo que se ha calificado como una atención residual contraria al espíritu de la Ley ${ }^{26}$. Por ejemplo, las madres solas con hijos a cargo no han contado con apoyos públicos suficientes lo que les ha venido ocasionando una situación de inseguridad y de permanente sacrificio, resultándoles casi imposible lograr compaginar trabajo y cuidado de los hijos, tal como se les exige ${ }^{27}$.

- Insuficiente apoyo a quienes prestan servicios a personas con discapacidades.

Los Departamentos de Servicios Sociales tienen obligación de realizar una evaluación de las necesidades de toda persona que es cuidada en la comunidad, incluyendo a quien realiza los cuidados. Sin embargo, parece que esto se ha cumplido en Salud Mental pero en otro casos sólo se ha cumplido por la demanda expresa de la persona con discapacidad.

- Problemas organizativos de los Servicios.

Hay quejas de falta de coordinación, falta de planificación y falta month.s. Leicester, Leicester University.

25 Social Services Inspectorate. Social Services Facing the Future, the 1997/98 annual report of the Chief Inspector.

26 Tunstill, J. (1995): «Children in need: Is targeting the Answer?», en Targeting those most in need: Winners and losers, Londres, Policy Studies Institute.

27 Van Drenth, A.; Knijn, T.; Lewis, J. (1999): "Sources of Income for Lone Mother Families: Policy Changes in Britain and The Netherlands and the Experiences of Divorced Women". Journal of Social Policy, 28 (4), 619-641 
de cualificación de muchas de las personas que prestan atención a los menores. En el primer caso, parece que se habla indistintamente de coordinación, colaboración y cooperación entre agencias, utilizándose erróneamente como sinónimos y, en realidad, limitándose a una mera cooperación ${ }^{28}$. Se habla mucho de trabajar conjuntamente pero, en la práctica, no se llega a realizar. Otras deficiencias señaladas son la tendencia a actuar con los menores con poco rigor, al detectarse falta de planificación y sistematización en las actuaciones, así como la necesidad de una formación especializada multidisciplinar que incluya conocimientos, valores y destrezas y de apoyo/soporte a los profesionales ${ }^{29}$. La efectividad de un buen trabajo con los menores exige la elaboración de políticas y procedimientos de actuación, además de facilitar los recursos necesarios y el apoyo a los profesionales ${ }^{30}$.

- Conflicto entre los roles asignados a los Trabajadores Sociales. Por una parte, están obligados por la ley a trabajar conjuntamente con los padres y, al mismo tiempo, defender los intereses del menor, por lo que tienen que jugar un rol de agentes de control social. El propio sistema limita la realización de un trabajo social que promueva la mayor participación de los padres y la atención a menores se ha dirigido fundamentalmente a clasificar el riesgo de los menores, registrándolos, antes que a atender las necesida$\operatorname{des}^{31}$.

- Falta de participación de familias y menores.

Se reconoce que es imprescindible considerar el rol central que juegan las familias y los propios menores ${ }^{32}$ y dar carácter prioritario a los servicios de apoyo a las familias, tanto para ayudarles en su tarea como para ofrecer a los padres la capacitación nece-

Department of Health (1991): The Children Act 1989. Guidance and Regulations: Volume 2. Family Support, Day Care and Educational Provision for Young Children. Londres, HMSO; Birchall, E.; Hallett, C. (1995): Working Together in Child Protection. Londres, HMSO. Thoburn, J.; Lewis, A. y Shemmings, D. (1995): Paternalism or Partnership? Families' hwvolvement in the Child Protection Process. Londres, HMSO. Horwath, J.; Calder, M. (1998): "Working Together to Protect Children on the Child Protection Register: Myth o Reality". British Journal of Social Work, 28, 879-895.

30 Fisher, T. (1995): Child Protection: What Knowledge Do Social Workers Use? York, The University of York, Social Work Research and Development Unit.

31 Bell, M. (1999): "Working in Partnership in Child Protection: The Conflicts". British Journal of Social Work, 29, 437-455.

32 Documentos oficiales que ofrecen orientaciones para este partenariado con las familias: Department of Health and Social Security (1995): The Challenge of Patemship. Para conocer "buenas prácticas"de participación de la familia en la conferencia inicial ver: Department of Health and Social Security (1995): Child Protection: Messages from Research. Londres, HMSO. 
saria para que puedan prestar una mejor atención a sus hijos.

- Inadecuación de las alternativas familiares asignadas a los menores.

Se ha elaborado un Libro Blanco sobre la Adopción ${ }^{34}$ que analiza la situación de los menores a cargo de los Councils en Inglaterra. El estudio destaca que, a finales de marzo de 1998, había unos 58.000 menores a cargo de los Councils ingleses, estimándose que un $40 \%$ de estos menores vuelven a su familia de origen en menos de 8 semanas y el $60 \%$ restante tiene dificultades en encontrar estabilidad familiar. De este segundo grupo de menores con dificultades, se estima que un $18 \%$ experimenta tres o más cambios de familia en un año; el $70 \%$ deja de estar al cuidado de los Councils a los 16 años sin ninguna cualificación; el $25 \%$ entre 14-16 años no acude regularmente a un centro escolar y muchos han sido excluidos y no disponen de una plaza escolar; entre el $14 \%$ y $25 \%$ de las jóvenes están embarazadas o tienen un niño mientras en la población general sólo el $3 \%$ de las jóvenes de 20 años tienen un niño; tienen 60 veces más posibilidades de ser homeless y de ser delincuentes el (39\% de los presos menores de 21 años). También se destaca que ha habido una gran heterogeneidad entre los Councils en el uso de la adopción, además de falta de consistencia en la Ley de Adopción (Adoption Act 1976) y falta de concordancia con la Ley del Menor; la insuficiente especialización de los trabajadores sociales y la falta de apoyo a los padres adoptantes.

\subsection{La reforma de Mr. Blair}

Desde 1997 el nuevo gobierno laborista de Mr. Blair está llevando a cabo importantes cambios en los Servicios Sociales, como parte de una estrategia más amplia de reorientación de la política socioeconómica según el nuevo modelo denominado «Tercera vía». Con relación a los servicios sociales, se ha elaborado un Libro Blanco y diversos documentos de evaluación que analizan la situación actual y marcan las nuevas orientaciones que han guiado las reformas en curso ${ }^{35}$.

La nueva política se basa en el mantenimiento del Estado de Bienestar, pero modificando la filosofía original y considerando que la ma-

34 Department of Health (2000): Adoption-a new approach, A White Paper. Londres, The Stationery Office.

35 Department of Health. Social Services. Achievement and Challenge. Londres, The Stationery Office; HM Treasury y Cabinet Office (1998): Modern public services for Britain: investing in reform. Londres, The Stationery Office. 
yoría de las personas pueden atender sus necesidades por sí mismas, o con ayuda de su familia, amigos y vecinos, por lo que la principal responsabilidad de la atención social recae en cada individuo y en las redes sociales de ayuda mutua. El nuevo rol de los servicios públicos es apoyar y proporcionar atención a quienes carecen de redes sociales, es decir, a las personas muy dependientes y vulnerables que están aisladas socialmente, pero siempre con el objetivo de promover la independencia de las personas mediante mejores servicios de prevención y rehabilitación, incentivando la salida del sistema asistencial.

Para ello se considera necesaria una reorientación de la formación de los Trabajadores sociales y de los propios Departamentos de Servicios Sociales, así como de las personas que trabajan para el Tercer sector, en el que se ha constatado que hay muchos trabajadores que carecen de una adecuada cualificación. En este sentido, está prevista la sustitución del "Central Council for Education and Training in Social Work" (CCETSW) por el "General Social Care Council" (GSCC), que asumiría las competencias de regulación de la formación de los Trabajadores Sociales en Inglaterra y Gales, además de establecer los estándares para los restantes profesionales de los servicios sociales y mantener un Registro que incluya a los profesionales que, demostrada su adecuada formación, queden autorizados a ejercer ${ }^{36}$.

Se ha comenzado una reorganización de los servicios sociales ${ }^{37}$, como parte de una estrategia a largo plazo, en la que se mantiene la tendencia a apoyar la intervención de la iniciativa privada en la provisión de servicios sociales, asegurando que se pondrán los medios para garantizar la eficacia y eficiencia de los servicios prestados, así como la participación directa de los usuarios de los servicios, tanto en la determinación de sus necesidades como en la manera de satisfacerlas.

Más específicamente, en el caso de la atención social a los menores, este gobierno ha asumido los problemas que hemos señalado anteriormente junto a otros detectados en diferentes informes de evaluación realizados tras las elecciones de 1997. Los responsables de los Departamentos de Educación y Empleo, Seguridad Social y Mujeres presentaron en mayo de 1998 el documento "Meeting the Childcare Challenge" ${ }^{\text {" } 38}$ en el que se identifican los problemas y se establecen las nuevas estrategias de acción. Los principales problemas señalados son tres:

36 "Care Standards Act 2000".

37 Department of Health (1998): Modernising Social Services. Pronoting independence, improving protection, raising standards. Londres, The Stationery Office.

38 Department for Education and Employment, Ministers for Women (1998): Meeting the Childcare Challenge. Londres, The Stationary Office. 
a) falta de regulación de los estándares de atención a los menores, por lo que la calidad de los cuidados es muy variable;

b) alto coste de los servicios, por lo que no están al alcance de muchos padres;

c) insuficiencia de plazas de atención a menores en algunas zonas y falta de información sobre la oferta existente.

La nueva estrategia pretende asegurar una atención de calidad y accesible (Working Families Tax Credit) a los menores de edades comprendidas entre los 0 y los 16 años, en cada comunidad, con el fin de promover el bienestar del menor ofreciendo igualdad de oportunidades para los padres, especialmente a las mujeres, y apoyo para conciliar la vida laboral y familiar. Para conseguirlo, deben trabajar conjuntamente (en partenariado) el gobierno central, las Autoridades locales, las entidades privadas y voluntarias que prestan servicios a los menores, empresarios, instituciones de enseñanza superior, escuelas, autoridades de salud, centros para alumnos con necesidades educativas especiales y padres.

Se destaca que los Servicios Sociales para menores no pueden considerarse separadamente del conjunto de servicios que las Autoridades locales y otras entidades ofrecen a los menores, por lo que se tiende a una atención más global, por ejemplo, integrando las actuaciones de Salud y Servicios Sociales (tal como ya se ha comenzado a hacer en el ámbito de la Salud Mental) ${ }^{39}$. Se promete mayor apoyo a la familia; reducir la exclusión social y la conducta anti-social de menores; y proporcionar a cada menor la oportunidad de tener una vida saludable, feliz y con éxito, con especial a los menores más vulnerables.

Para subsanar los problemas identificados, se han establecido tres prioridades nacionales para los nuevos servicios sociales de atención a los menores:

1. Asegurar que los menores están protegidos de maltrato físico y emocional, abuso sexual o negligencia.

2. Mejorar la calidad de la atención a los menores en protección de la forma más parecida a como lo harían unos padres responsables.

3. Aumentar las oportunidades de vida de los menores en protección y de otros menores ("en necesidad") que necesiten apoyo

Esto ha supuesto muchas críticas por considerar que más allá de la unificación de recursos financieros supone un cambio de filosofía que se califica de retroceso al pasado en cuanto puede derivar en una "medicalización" de la atención a menores (tratamientos terapéuticos). 
de los servicios sociales, en particular, mejorando su salud y educación y apoyo cuando dejen de estar en situación de protección.

Como ejemplos para lograr estos objetivos se pueden citar planes de actuación como "Sure Start" (proporcionar oportunidades a menores de áreas sociales en desventaja a través de servicios de salud, educación, servicios sociales y organizaciones voluntarias) y "Early Years Development and Childcare Plans" (Plan integral de educación y atención social para menores y sus familias).

Y se están realizando numerosos cambios legislativos entre los que cabe destacar los siguientes:

- Reforma del sistema de protección social (prestaciones económicas).

La "Welfare Reform and Pensions Act 1999" reforma el sistema de pensiones y subsidios aplicando el nuevo principio de "trabajo para los que pueden y seguridad para los que no pueden" (Welfare to Work). Además, la "Child Support, Pensions And Social Security Act 2000" reforma el sistema de prestaciones por menores a cargo iniciado en 1991 (Child Support Act 1991 y Child Support Act 1995), modificando el procedimiento establecido para los cálculos de las cuantías de las prestaciones, teniendo en cuenta determinados costes extras por el mantenimiento del menor. Por ejemplo, se regulan las responsabilidades de los padres en la obligación de mantenerlos (incluyendo la presunción de paternidad); la relación entre el sistema de prestaciones por menor a cargo y las ordenes judiciales de alimentos; y la introducción de tarifas por los servicios de apoyo a menores en determinados supuestos.

- Reformas en el ámbito de la Administración Local.

La "Local Government Act 2000" asigna un nuevo rol a las Autoridades locales al encomendarles el desarrollo de estrategias comunitarias (en partenariado) con la finalidad de mejorar la calidad de vida de las comunidades locales, mediante la promoción del bienestar económico, social y ambiental de cada territorio. Se establece una nueva financiación para servicios asistenciales para las personas más vulnerables y se autoriza a cobrar por los servicios de cuidado de menores que no estén bajo la custodia de las Autoridades Locales y cuyos padres sean beneficiarios de "Working Families'Tax Credit" y "Disabled Person's Tax Credit". 
- Establecimiento de unos estándares básicos obligatorios. La "Care Standards Act 2000" establece la reforma del sistema de servicios de cuidados proporcionados por entidades privadas y voluntarias en Inglaterra y Gales. Se crea una Comisión Nacional que establecerá los estándares mínimos de calidad exigibles (National Care Standards Commission) en Inglaterra y se regula una amplia gama de servicios incluyendo desde la atención residencial hasta la ayuda a domicilio, el acogimiento familiar y la adopción (care homes, nursing homes, children's homes, domiciliary care agencies, fostering agencies, voluntary adoption agencies). Estos mismos estándares serán de obligado cumplimiento para los servicios públicos locales.

- Ampliación de la edad de protección a los menores y asignación de nuevas responsabilidades a las Autoridades Locales.

La "Children (Leaving Care) Act 2000" asigna a las Autoridades Locales mayores responsabilidades en el cuidado de los menores y jóvenes a su cargo que van a dejar de estar bajo protección, para lo que deben brindarles mayores oportunidades y prepararles para que puedan llevar a cabo una vida independiente. Como parte de estas nuevas responsabilidades deben ocuparse de que los jóvenes más vulnerables tengan la educación y formación ocupacional que les capacite para emplearse, incluyendo educación superior (con alojamiento y manutención). Los menores pueden abandonar el hogar a los 16 años pero, si es necesario, se amplía la edad de atención hasta los 21 años, para facilitar la transición desde el sistema de cuidados a la vida independiente.

- Prestaciones de apoyo a las personas que cuidan a personas dependientes.

La "Carers and Disabled Children Act 2000" encomienda a las Autoridades Locales la prestación de servicios de apoyo (prestación directa o pago por servicios) a las personas de 16 años o más que cuidan a personas con enfermedades de larga duración o con discapacidades (uno de cada seis hogares tiene una persona cuidadora). La ley establece y regula el derecho de las personas cuidadoras a recibir ayuda física, asesoramiento, formación y cortos descansos. Se establece que los servicios de apoyo deben ser flexibles para adecuarse a las diferentes situaciones concretas, teniendo en cuenta las preferencias de las personas interesadas (cuidadores y cuidados) y que se puede compensar económicamente o establecer un precio por los servicios. La ley también regula el derecho de las personas que tienen a su cargo 
a un menor con discapacidad a recibir por parte de las autoridades locales servicios de apoyo en su calidad de cuidadores.

\section{SERVICIOS SOCIALES PARA LOS MENORES Y SUS FAMI- LIAS}

La atención pública a los menores es una obligación que atañe a varios Departamentos Ministeriales. El Departamento de Seguridad Social es el responsable de una serie de prestaciones económicas para los padres que tienen la finalidad de compensarles los gastos ocasionados por los menores a su cargo. Las reformas recientes en la línea de que trabaje todo el que pueda vinculan las prestaciones a la aceptación de un trabajo, incluyendo a las madres solas con menores a cargo, tal como se ha comentado, quedando regulado en la "Child Support, Pensions and Social Security Act 2000"40. El Departamento de Salud, además de los servicios sociales en sentido estricto, proporciona servicios de protección materno-infantil a través de los Departamentos de Servicios Sociales locales; y el Departamento de Educación y Empleo proporcionan servicios de pre-escolar y extra-escolar, además de oportunidades de formación ocupacional y empleo.

En cuanto a los servicios de cuidado directo a los menores se pueden agrupar en tres grandes grupos ${ }^{41}$ :"Nursery education" (pre-escolar que se rige por la normativa del sistema educativo, Ley de Educación), "Childcare" (cuidados diurnos para hijos de padres trabajadores), "Welfare care" (para menores en riesgo o necesitados de protección). Las tres modalidades de actuación puede ofrecerse en un mismo lugar por autoridades locales, organizaciones voluntarias o entidades privadas y están siendo revisadas en el contexto de la reforma comentada.

a) La mayoría de los niños de 4 años cursan preescolar escolarizados en centros de educación primaria y se está escolarizando a los niños de 3 años $^{42}$. Además del aspecto cuantitativo de la escolari-

40 Un ejemplo de la reforma en este ámbito es la "Working Families'Tax Credit". Puede encontrarsc información detallada sobre la protección social de la Seguridad Social en www.dss.gov.uk

4) Penn, H. (2000): "Policy and Practice in Childcare and Nursery Education". Journal of Social Policy, 29 (1), 37-54.

42 Siguiendo la experiencia de otros países europeos en los que los menores de tres/cuatro años reciben atención integral (cuidado y educación) en centros del sistema educativo ordinario. European Commission (1996): A review of services for young children in the European Union 1990-1995. Bruselas, European Commission Childcare Network, European Commission DG $\mathrm{V}$. 
zación, se está trabajando en el tema de la calidad de los servicios ofrecidos ${ }^{43}$.

b) El Informe "Meeting the Childcare Challenge" destaca la necesidad de proporcionar más plazas para padres trabajadores, como apoyo a la política de "Welfare to Work" que ofrece subsidios para pago de hasta el $70 \%$ de los servicios de cuidado de los menores a los padres trabajadores con bajos ingresos. Un 90\% de los servicios de pre-escolar y cuidados de día se prestan por la iniciativa privada mercantil. La nueva ley "Care Standards Act 2000" transfiere la responsabilidad de estos servicios de cuidado diurno de menores, hasta ahora a cargo de las Autoridades locales de Educación, al Servicio de Inspección Escolar (Her Majesty's Chief Inspector of Schools for England -HMCIS).

c) Welfare care. Entre otras medidas de lucha contra la exclusión social, se ha puesto en marcha un programa específico de intervención temprana dirigido a menores de tres años pertenecientes a familias con problemas. Se trata de trabajar con los padres para promover el desarrollo físico, intelectual y social de los menores y prevenir el fracaso escolar. También se ofrecen servicios de recreo, salud, orientación y apoyo a los padres, así como otros necesarios para satisfacer sus necesidades.

\subsection{La Ley de Menores de 1989 (Children Act 1989)}

Esta ley, que continúa en vigor, introdujo importantes cambios en la política de protección de menores, reorganizando el reparto de las responsabilidades entre Administración, familias y menores y asegurando atención a todo niño «en situación de necesidad». La ley establece la obligación de los Departamentos de Servicios Sociales locales de elaborar planes trienales de su oferta de servicios de cuidado de los menores (Children's Services Plans) ${ }^{44}$.

Entre los motivos que se adujeron para el cambio de legislación se señalaba que la anterior Ley de Menores ponía el énfasis en los derechos y obligaciones de los padres y, en la práctica, no garantizaba los

European Commission (1997): Quality Targets in Services for Young European Children. Bruselas, European Commission Childcare Network, European Commission DG V.

Este concepto «en necesidad» se ha relacionado tradicionalmente con situaciones de pobreza y privación material (niños «pobres») y se ha abusado de su separación del entorno familiar. La nueva ley reconsidera esta medida, restringiéndola al máximo y reconociendo también las necesidades de los padres. Sin embargo, los Trabajadores Sociales se quejan de que, en la práctica, se sigue sin trabajar para prevenir el abuso, respondiendo sólo a situaciones de crisis y a los requerimientos del procedimiento legal ante el abuso o maltrato de un menor. 
derechos del menor, considerándose que los Departamentos de Servicios Sociales locales actuaban sólo ante situaciones de abuso de los menores y no en su prevención. Ante una posible situación de maltrato de un menor la solución más común consistía en alejarle de su familia, adoptando los trabajadores sociales un rol de control social al cumplir su función de defender al menor sin tener en cuenta a la familia. La experiencia demostró que las actuaciones con los menores separados de sus familias no garantizaban su bienestar ya que, por una parte, muchos de ellos habían sido objeto de maltrato y abuso en sus nuevos alojamientos y, por otra, gran número de estos menores al cumplir la mayoría de edad no estaban en condiciones de poder vivir de forma independiente. Todo ello puso de manifiesto la carencia o inadecuada atención que se prestaba a los menores, por parte de los Departamentos de Servicios Sociales, tras separarles de su familia, originándose un debate nacional sobre la política de atención a los menores ${ }^{45}$.

En consecuencia, la Ley de Menores de 1989 trata de encontrar el equilibrio entre los derechos de los padres y los derechos de los hijos, siendo el objetivo general el de salvaguardar y promover «el mejor interés del menor» hasta que cumpla los 18 años, asignando a las autoridades locales la responsabilidad de promover el Bienestar del menor, contando con el punto de vista de éste y designándole un tutor o representante cuando sea necesario. Como novedades cabe destacar el concepto de «responsabilidad parental» y la aplicación del principio de presunción de «no-intervención» en la vida familiar si no es necesario, además del reconocimiento de la necesidad de un «partenariado», es decir, de establecer una cooperación estrecha familia-profesionales de los Servicios Sociales trabajando desde una relación de igualdad ${ }^{46}$. Se reconoce así que los padres son responsables del cuidado de sus hijos y que los poderes públicos no deben intervenir en la vida de una familia, salvo que sea estrictamente necesario. En el caso de que se deba intervenir, no se puede hacer al margen de la familia, por el contrario, el punto de partida es que la familia suele ser la mejor opción para el menor, por lo que hay que trabajar con la familia para solucionar los

45 "Sccond Report of the House of Commons Social Service Select Committee" (198384): "White Paper on The Law on Child Care and Family Services" (1987). El estudio con mayor repercusión social fue el Informe sobre el caso Cleveland (1988) que denunciaba la desconsideración hacia los menores, a los que se trataba como a meros objetos o como problemas sociales. «Report of Inquiry into Child Abuse in Cleveland» $(\mathrm{Cm}$ 412). Londres, HMSO.

46 Department of Health (1991): The Children Act 1989. Guidance and Regulations. Volume 3. Family Placements. Londres, HMSO. 
posibles obstáculos en el desarrollo del menor, incluso si se separa de ella al menor. ${ }^{47}$

Por «responsabilidad parental» se entiende todos los derechos, deberes, poder, responsabilidades y autoridad que por Ley tienen el padre de un menor en relación con el menor y sus bienes y sólo el juez puede determinar si los padres pierden esta responsabilidad parental. La madre nunca pierde la responsablidad parental, aunque una Orden de Cuidado hace que la comparta con la Autoridad Local. El padre casado legalmente con la madre del menor adquiere automáticamente la responsabilidad parental y, si no hay unión legal, la madre puede solicitarla para él. Los padres pueden perder su responsabilidad parental cuando un juez decida que el menor se encuentra en «situación de necesidad» ${ }^{48}$.

Según la ley, un menor puede encontrarse «en necesidad» en los siguientes supuestos:

a) Si de manera indudable no puede alcanzar un estado de bienestar aceptable sin que se le proporcionen cuidados por la Autoridad Local.

b) Si su salud o su desarrollo puede ser «significativamente» perjudicados si no se le proporcionan cuidados.

c) Si es valorado como discapacitado de acuerdo con la Ley de Menores 1989.

Otro concepto clave es el de «daño significante». La ley considera que hay tal cuando el daño sufrido por el menor, en relación con su salud y desarrollo, es mayor del que razonablemente se puede esperar con relación a un menor similar y, en caso de abuso físico, las circunstancias del daño pueden ser tan significativas como los daños en sí mismos. Las categorías del maltrato son: negligencia, maltrato físico, abuso sexual y maltrato emocional o psicológico.

Se considera crucial la cooperación de los distintos Departamentos de la Autoridad Local (Salud, Vivienda, Educación y Servicios Sociales) para proporcionar un servicio integrado así como con las Agencias, estando todos ellos obligados a apoyar a los responsables del cuidado de los menores (sean sus padres, parientes o familias sustitutas) con información, preparación, y los necesarios servicios de día ${ }^{49}$.

47 En la práctica, hay fuertes presiones para separar al menor de su familia. Una corriente de Trabajadores Sociales lo considera una forma de abuso profesional.

48 Los jueces suelen dictar una Orden de Cuidados sólo en caso de abuso y raramente en caso de maltrato psicológico por la dificultad de acreditarlo suficientemente.

49 Department of Health (1991): The Care of Children. Principles and Practice in Regulations and Guidance. Londres, HMSO.(4a edic.), pág. 12-14. 989). Sin embargo, 
Las obligaciones específicas de los Departamentos de Servicios Sociales implican desde la provisión de servicios de prevención y atención al menor, incluyendo un alojamiento alternativo a su familia, hasta el inicio de procedimientos de protección del menor cuando los servicios no pueden garantizar el bienestar del menor, tal como se describe a continuación.

\subsection{El procedimiento de protección de los menores}

La ley establece que los Trabajadores sociales deben investigar los casos de maltrato de menores y realizar una investigación permanente de los menores en situación de riesgo inscritos en un Registro al efecto. Las situaciones de maltrato de los menores se conocen en los Departamentos de Servicios Sociales, fundamentalmente, a través de los centros escolares y también por denuncias de ciudadanos, policía, profesionales de la salud e, incluso, por miembros de la familia del menor. La investigación con relación al maltrato de un menor debe realizarse conjuntamente por los Departamentos de Servicios Sociales y la policía, que cuenta con profesionales especialmente entrenados para ello. Además, deben colaborar en la investigación otros Departamentos de la Administración Local como Educación, Vivienda y Salud.

Los Trabajadores sociales de los Departamentos de Servicios Sociales tienen la obligación legal de investigar para determinar las necesidades del menor y poder actuar en las siguientes circunstancias:

- Cuando hay sospecha fundada de que un menor que vive o se encuentra en su área está sufriendo o parece sufrir daño significante.

- Cuando hay bajo una Orden de Protección de Emergencia (con intervención del Juzgado) o una Orden de Protección Policial (sin intervención del Juzgado).

- Cuando un Juzgado de Familia solicita que investiguen las circunstancias del menor.

- Cuando el Departamento de Educación les notifica que un menor tiene problemas.

Esta investigación tiene como finalidad evaluar el bienestar del menor, valorando la conveniencia de que se inicien los procedimientos para que quede bajo la protección y cuidado de los Departamentos de Servicios Sociales locales. Cuando esto último ocurre, se comparte la «res-

en la práctica, difícilmente se cumple. Por ejemplo, cuando los Trabajadores Socialcs consideran que es importante otra vivienda para el bienestar del menor y, así lo solicitan, no suelen encontrar respuesta. 
ponsabilidad parental» con los padres biológicos y puede durar hasta que el menor cumple los 18 años, estableciendo el juez el régimen de contactos y visitas entre el menor y su familia. Los contactos y encuentros entre un menor y otra persona incluyen: visitas, estancias, salidas y comunicación por carta, teléfono y similares. La Autoridad local debe permitir al menor (bajo su cuidado) contactos razonables con un determinado número de personas, incluidos sus padres, determinando el juez su naturaleza y frecuencia, que siempre se basará en el beneficio del menor y no en el de los adultos.

La ley obliga a que en cada Distrito haya un "Registro de Protección del Menor" que ha sufrido maltrato o que se considera en riesgo (Child Protection Register) en el que se centraliza la información existente sobre protección de menores, quedando obligados los profesionales que trabajan con los menores a elaborar documentación e información sobre las situaciones de maltrato infantil. Este Registro es estrictamente confidencial y debe estar siempre accesible para ser consultado. El Informe del menor debe contener la siguiente información: identificación del menor y la familia, así como otros detalles de otros niños en el hogar; la naturaleza del maltrato; el trabajador de referencia y el «core group $»^{50}$; el trabajador social general; el visitador de salud; el nombre de la escuela; personas de referencia; investigaciones, planes de tratamiento, seguimiento y revisiones periódicas.

Para tomar las decisiones, hay unas Comisiones de evaluación de casos (Child Protection Case Conferences) a cuyas reuniones se invita a los padres a participar y en los que los diferentes profesionales implicados en un caso intercambian información, coordinan sus acciones en referencia a un menor y su familia y deciden conjuntamente inscribir o no al menor en el Registro, valorando si hay daño significante para que sea necesario un plan de protección del menor. Periódicamente se revisa y valora la permanencia de los menores en el Registro. La decisión sobre el alejamiento del menor de su familia compete al juez.

También en cada área hay un «Comité de Protección del Menor» (Area Child Protection Committee -ACPC-), formado por un grupo interdisciplinar de profesionales que representan a cada entidad implicada en la protección del menor: Educación, Salud, Servicios Sociales. Su función es elaborar, controlar y evaluar las políticas y la práctica del

50

Cuando un menor queda incluido en el Registro de Protección del Menor, se nombra un Trabajador Social de referencia que se encarga de coordinar el trabajo con la familia y visitarla regularmente. También se nombra un "Core Group" o pequeño grupo de profesionales que elaboran juntamente con la familia un Plan de Protección del Menor (Child Protection Plan) y deciden quién hará qué y cuándo, reuniéndose periódicamente para llevar a cabo un seguimiento del Plan. 
trabajo en el campo de los menores en su área, coordinando las actuaciones de todos los profesionales que intervengan.

En algunos lugares se sigue el modelo de «Family Group Conference» en el que los miembros de la familia tienen la responsabilidad de decidir cómo proteger mejor al menor, participando el menor y muchos miembros de la familia extensa así como otros miembros de la comunidad que van a poder ser de ayuda para el menor en el futuro. Los profesionales de los servicios sociales están presentes para orientar y ayudar a tomar decisiones, pero las decisiones las toma la fami$\mathrm{lia}^{51}$.

También existe la posibilidad de que los menores en riesgo puedan pasar al cuidado de la Autoridad Local sin medidas judiciales, siempre que así lo acuerden los padres. Se nombra un «Guardian-ad-litem»o trabajador social independiente para asegurar que se vela por los intereses del menor.

En cuanto a la duración de la protección, la Autoridad Local tiene la obligación de cuidar del menor durante el tiempo que dure la Orden de Cuidados, hasta que el menor cumpla los 18 años o hasta que esta Orden se recurra para ser revocada, o se otorgue una Orden de Residencia a favor de un familiar del menor que se ofrece a cuidar de él para evitar un acogimiento en familia extraña o un ingreso institucional y el Juez le otorga la responsabilidad parental. El sistema de protección puede continuar cuando los menores cumplen los 18 años si no son capaces de mantenerse de forma independiente, en estos casos, las Autoridades locales siguen teniendo obligación de orientación y cuidados por parte de la Autoridad local hasta los 21 años, mediante ayudas para alojamiento, salud, ingresos, trabajo y formación.

2.3. Servicios para Menores que se ofrecen desde los Departamentos de Servicios Sociales locales.

Las obligaciones que la ley asigna a los Departamentos de Servicios Sociales de las Autoridades Locales son las siguientes:

a) Supervisar al menor atendido en guarderías privadas, play-groups y childminders.

b) Prevenir la necesidad de tomar bajo tutela al menor o que llegue a un Tribunal Juvenil.

c) Tomar bajo tutela a los menores cuyos padres demuestren su incapacidad de cuidarlos. 
d) Actuar a través de los Tribunales para velar por los intereses del menor y del joven cuando sean necesarias acciones legales.

e) Velar por los intereses de los menores bajo tutela y proporcionarles alojamiento y manutención.

f) Supervisar al menor que va a ser adoptado y acordar el lugar para la adopción teniendo en cuenta el mejor interés del menor.

g) Supervisar al menor que está viviendo en Acogimiento familiar.

Como parte de estas obligaciones, las Autoridades Locales deben proporcionar apoyo a las familias ofreciendo diferentes servicios, bien directamente o bien a través de entidades privadas (tanto mercantiles como sin ánimo de lucro), por ejemplo, orientación y atención psicológica; actividades ocupacionales, sociales, culturales o recreacionales; ayuda a domicilio, incluyendo lavandería; atención a itinerantes; atención a menores con discapacidades; vacaciones para menores y familias. Cuando el menor no pueda convivir con su propia familia se buscará la mejor alternativa, siempre contando con sus deseos y estableciendo un acuerdo con su familia. Las alternativas son una familia de acogida, alojamiento residencial o adopción.

Cada Autoridad local puede establecer su oferta de servicios para cumplir con estas obligaciones. A continuación, de modo muy sintético, se comentan algunas de las características de la atención a menores mediante Acogimiento familiar, Adopción y Centros de familia, así como un breve comentario sobre las líneas básicas de atención a menores con discapacidades, a menores de familias "sin techo", prevención de drogodependencias y atención a menores con problemas legales.

\section{- Acogimiento Familiar.}

Los Departamentos de Servicios Sociales deciden qué menores deben pasar a la situación de acogimiento, pero la prestación del servicio se puede encomendar a entidades privadas que son las responsables de seleccionar y formar a las familias acogedoras así como del seguimiento correspondiente. Los Trabajadores Sociales de los Departamentos de Servicios Sociales deben supervisar el acogimiento y velar por el bienestar del menor, intentando que retorne a su propia familia, siendo la reunificación familiar, y en el más corto tiempo posible, la primera meta de los acogimientos familiares. La ley obliga a tener en cuenta las diferencias debidas a raza, cultura y lengua de los menores. El acogimiento puede considerarse una "actividad profesional" y estar remunerado por los Departamentos de Servicios Sociales exigiendo una cualificación y experiencia en el cuidado de menores y que una figura parental tenga disponibilidad para ocuparse a tiempo completo del cuidado del 
menor. Otras Autoridades Locales, por ejemplo, Doncaster, han preferido optar por familias acogedoras voluntarias de la propia comunidad a las que proporcionan formación para que cuiden adecuadamente al menor y una compensación por los gastos de manutención del menor.

\section{- Adopción}

Hay equipos con Trabajadores sociales especializados en Adopción y se están introduciendo cambios importantes para mejorar los procedimientos hasta ahora habituales siguiendo las orientaciones del citado Libro Blanco sobre Adopción. El gobiemo se propone incrementar el número de adopciones modificando la legislación y colocando las necesidades y los derechos del menor en el centro del proceso. Los cambios se dirigen a establecer nuevos procedimientos en la captación, formación y apoyo a los padres adoptantes; mejoras en los servicios de adopción de los servicios sociales locales ${ }^{52}$; mejoras en el sistema judicial relativo a la adopción e, incluso, cambios legislativos en la línea de la Ley del Menor de 1989.

Entre estos cambios legislativos se propone una nueva modalidad de convivencia alternativa a la familia de origen para los casos en los que un menor no puede retornar a su familia pero tampoco la adopción resulta una opción adecuada, por ejemplo, en el caso de algunas minorías culturales o religiosas. Esta nueva figura se denominaría "special guardianship" y proporcionaría a la persona que se hiciera cargo del menor la responsabilidad de su cuidado, convivencia estable, seguridad legal, vínculos legales entre el menor y su familia de origen y acceso a todo tipo de servicios de apoyo a la familia, incluyendo apoyo económico.

En cuanto a la Adopción internacional, la "Adoption (Intercountry Aspects) Act 1999". regula la adopción internacional adaptando la Ley de Adopción ("Adoption Act 1976" para Inglaterra y Gales y "Adoption Act 1978" para Escocia), facultando a las Autoridades locales (Departamentos de Servicios Sociales locales) y a las Agencias privadas autorizadas por el Secretario de Estado.

52 Incluyendo mayor formación especializada para los trabajadores sociales que trabajan con menores. El Department of Health y el Central Council for Education and Trainig in Social Work han creado un nueva titulación de post-grado "Post-Qualifying Award in Child Care", instando a los Departamentos de Servicios Sociales locales para que faciliten a sus trabajadores sociales su obtención. 


\section{- Centros de Familia}

Se atiende a los menores, a sus familias o cuidadores proporcionándoles también actividades ocupacionales, sociales, culturales o recreacionales y orientación/atención psicológica, además de servir de centros de atención diurna de los menores. El Centro de Familia es un Centro de día, con la peculiaridad de que se ofrece ayuda intensiva a una parte o a toda la familia, cuando ésta tiene graves problemas. Los menores acuden en régimen de media pensión y se proporciona formación a los padres para que aprendan a prestar los cuidados adecuados a sus hijos en las actividades de la vida cotidiana, de este modo se promueve la permanencia de los menores en su propio hogar. Los padres pueden ser legalmente obligados a acudir para recibir educación familiar y capacitación para poder satisfacer las necesidades físicas, emocionales y sociales de sus hijos. Es un recurso que puede tener también un carácter preventivo, reduciendo las probabilidades de problemas familiares graves. Se trabaja en coordinación con los Trabajadores Sociales de los Departamentos de Servicios Sociales y de otras entidades sociales de la zona. Estos Centros pueden ofrecer una variada gama de prestaciones trabajando simultáneamente con unos 20 niños de los Registros de Protección de Menores. Se suele incluir la oferta de un club con horario extraescolar, ludoteca, etc. Hay especialistas de apoyo que pueden acudir regularmente al Centro, como logopedas, enfermeras, oficiales de probation, abogados de familia, etc. También se puede utilizar el Centro para grupos de adolescentes u otras necesidades de la comunidad, como lugar de reunión y actividades de las entidades públicas y sociales de la comunidad.

\section{- Atención a menores con discapacidades.}

Como ya se ha comentado, la Children Act de 1989 define a los menores con discapacidades entre las categorías de «menores en necesidad» y establece que los Departamentos de Servicios Sociales locales deben ofrecer servicios a estos menores para reducir los efectos de su discapacidad y darles la oportunidad de vivir una vida tan normal como sea posible (prevención). Se considera que estos menores tienen las mismas necesidades que cualquier otro menor y, además, una serie de necesidades especiales en función de su discapacidad. Por lo tanto, tienen derecho tanto a beneficiarse de los servicios para menores como a recibir ayuda específica complementaria para minimizar las consecuencias de la discapacidad, mejorar su calidad de vida y promover su independencia.

Suele haber Trabajadores Sociales especializados (Children with 
Disabilities Teams) que trabajan en cooperación con otras entidades, con los menores y con sus familias para asegurar una atención integral al menor, realizando, entre otras, las siguientes actuaciones: identificar e incluir en el Registro de menores en riesgo a los menores con discapacidad; evaluar sus necesidades y las de su familia; garantizar y promover el bienestar del menor de acuerdo con los padres y el propio menor; trabajar en cooperación con otras entidades; tener en cuenta los mismos principios generales que la ley señala para los menores en protección y los mismos servicios (integración).

Los padres de menores con discapacidades tienen derecho a nuevos servicios de apoyo en su calidad de cuidadores, en aplicación de la "Carers and Disabled Children Act 2000", tal como ya se ha mencionado.

- Atención a menores de familias "sin techo"

Hay Trabajadores Sociales que trabajan con "Homeless Families". Se les suele ofrecer alojamiento en la modalidad de «Bed \& Breakfast» (alojamiento y desayuno) o un Albergue (Hostel). A estos menores se les proporcionan servicios socioeducativos, como «School Holiday Play Schemes», con programas que proporcionan oportunidades de juego supervisado para menores de 2 a 5 años. A los padres se les estimula a participar activamente en la mayoría de los programas. Hay programas coparticipados por los Departamentos de Salud, Servicios Sociales y Educación, que se llevan a cabo en la Escuela y también se trabaja en coordinación con el sector voluntario.

- Prevención de drogodependencias, dirigida a menores entre 11 y 18 años de edad.

Son Programas interdepartamentales en los que trabajan de modo integrado profesionales de la Policía, servicios de trabajo con jóvenes ("Youth and Commnunity y Youth Offending Teams"), servicios de tratamiento de drogodependencias, servicios de salud y servicios de la prisión. Se realizan intervenciones en las escuelas y en la comunidad, ofreciendo información y apoyo a los jóvenes/sus padres y luchando contra el absentismo escolar como medida de prevención de la exclusión social.

- Los Departamentos de Servicios Sociales también se ocupan del Registro, asesoramiento, formación y supervisión de una serie de servicios para menores de 8 años como, por ejemplo:

- «Chilminders»: Son cuidadoras de niños que cuidan en su propio 
domicilio a uno o varios menores a su cargo.

- «Playgroups»: Actividades socioeducativas para niños de edad pre-escolar (0-5).

Hay childminders y playgroups especializados en la atención a menores «en necesidad», con riesgo de maltrato o en situaciones de ruptura familiar.

- «Creches»: son guarderías en las que se cuida a los menores entre 0-5 años, con atención diferenciada de 0-6 meses y de 6 meses a 5 años.

- «Out of School Clubs» y «School holiday play scheme» (2-5 años) en los que se realizan actividades socioeducativas durante tiempo libre y vacaciones.

- "Adventure play grounds", donde el niño juega con otros niños bajo la supervisión de adultos.

- «Nursery School»: guarderías privadas (0-5).

Estos servicios pueden ser públicos o privados (de iniciativa social o lucrativos). Si son privados, los servicios sociales locales tienen la obligación de inspeccionarlos para asegurase que cumplen los estándares establecidos en la legislación. También deben mantener un Registro de estos servicios y childminders. El Departamento de Empleo cofinancia algunos de estos servicios (tres años) para dar más oportunidades a las madres a aceptar un empleo.

- Atención a menores y jóvenes delincuentes.

Los "Youth Offender Teams" son equipos pluridisciplinares especializados en el trabajo con jóvenes delincuentes que tienen como finalidad coordinar las actuaciones concernientes a cada joven. Ha habido recientes modificaciones con la aprobación de dos normas legales, la "Crime and Disorder Act 1998" y la "Youth Justice and Criminal Evidence Act 1999", que establecen la reforma del sistema de justicia juvenil en Inglaterra y Gales, tratando de aplicar las conclusiones del Libro Blanco "No More Excuses" (CM 3809 Noviembre 1997), en el que se proponían mejoras en los tribunales juveniles de justicia y en la prevención de la delincuencia de menores y jóvenes.

La filosofía es la de tratar al joven delincuente no como a un criminal sino como a un joven que necesita ayuda social, aplicando una especial consideración cuando un menor de 18 años delinque por primera vez con la finalidad de prevenir su reincidencia. El "Youth Offender Panel" es el encargado de establecer un programa de conducta consensuado con el joven, que debe incluir un elemento de reparación 
del daño causado a todas las personas afectadas, si ellos lo consienten. El programa debe incluir diversas actividades, dependiendo de las circunstancias, por ejemplo: sesiones de mediación; trabajo no remunerado a favor de la comunidad; asistencia a un centro escolar; participación en actividades específicas como formación ocupacional o tratamiento de adicciones, etc. En cuanto al procedimiento a seguir, cabe destacar las siguientes actuaciones: evaluación de las necesidades del joven; programas de apoyo para los jóvenes en libertad provisional; acogida en establecimientos o en acogimiento familiar especializado o en medio cerrado; realización de los informes establecidos por los Tribunales, incluyendo la propuesta de la pena; orientación y ayuda a los jóvenes durante y después de la condena.

En conclusión, los Servicios Sociales de atención a menores en Inglaterra están inmersos en una situación de cambio dentro de un contexto de cambio más amplio, que afecta tanto al propio sistema general de Servicios Sociales como a otras áreas directamente relacionadas con el bienestar de las personas. En realidad, se está redefiniendo el propio modelo de Estado de Bienestar, por lo que los cambios han de valorarse desde una perspectiva de globalidad, tanto en su vertiente filosófica como en el ámbito de la práctica ya que, como se ha podido ver, estos cambios también tienen repercusiones en la formación de los profesionales de la atención social, incluyendo a los Trabajadores Sociales. Estamos asistiendo a un proceso que acaba de comenzar, por lo que habrá que seguir de cerca su desarrollo, no sólo por "curiosidad" científica sino, también, porque, como miembros de la Unión Europea, no nos puede resultar indiferente. 